\title{
Newly appointed editor of Archiv der Mathematik
}

\author{
BenJAMin KLOPSCH
}

Benjamin Klopsch, born 1974, studied mathematics and physics in Germany and in the UK. After completing his DPhil at the University of Oxford in 1999, he held postdoctoral fellowships in Israel and in Canada. Subsequently he worked as a Wissenschaftlicher Assistent at the University of Düsseldorf in Germany, obtaining his Habilitation in 2006. In 2007 he took up a lectureship at Royal Holloway, University of London, UK, where he was successively promoted up to the rank of a professor. In 2012 he accepted a professorship at the University of Magdeburg in Germany. Since 2013 he is a professor at the Heinrich-Heine-University in Düsseldorf, heading the algebra and number theory group.

His research is centred around group theory and group actions, with a view toward arithmetic, combinatorial, geometric and representation-theoretic aspects. He takes a keen interest in p-adic Lie groups, general locally compact groups and their lattices.

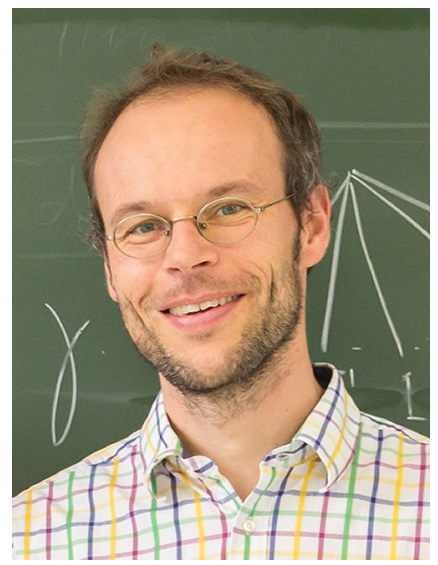

Benjamin KLOPSCH

Mathematisches Institut,

Heinrich-Heine-Universität Düsseldorf,

40225 Düsseldorf, Germany

e-mail: klopsch@math.uni-duesseldorf.de 